\title{
Functional interaction between Cdc42 and the stress MAPK signaling pathway during the regulation of fission yeast polarized growth
}

\author{
Pilar Pérez $^{1} \cdot$ Teresa Soto $^{2} \cdot$ Elisa Gómez-Gil $^{2} \cdot$ Jose Cansado $^{2}$ (D)
}

Received: 1 February 2019/Revised: 18 March 2019 / Accepted: 20 March 2019 / Published online: 15 April 2019

(C) Springer Nature Switzerland AG 2019

\begin{abstract}
Cell polarization can be defined as the generation and maintenance of directional cellular organization. The spatial distribution and protein or lipid composition of the cell are not symmetric but organized in specialized domains which allow cells to grow and acquire a certain shape that is closely linked to their physiological function. The establishment and maintenance of polarized growth requires the coordination of diverse processes including cytoskeletal dynamics, membrane trafficking, and signaling cascade regulation. Some of the major players involved in the selection and maintenance of sites for polarized growth are Rho GTPases, which recognize the polarization site and transmit the signal to regulatory proteins of the cytoskeleton. Additionally, cytoskeletal organization, polarized secretion, and endocytosis are controlled by signaling pathways including those mediated by mitogen-activated protein kinases (MAPKs). Rho GTPases and the MAPK signaling pathways are strongly conserved from yeast to mammals, suggesting that the basic mechanisms of polarized growth have been maintained throughout evolution. For this reason, the study of how polarized growth is established and regulated in simple organisms such as the fission yeast Schizosaccharomyces pombe has contributed to broaden our knowledge about these processes in multicellular organisms. We review here the function of the Cdc42 GTPase and the stress activated MAPK (SAPK) signaling pathways during fission yeast polarized growth, and discuss the relevance of the crosstalk between both pathways.
\end{abstract}

Keywords GTPase $\cdot$ Cdc42 $\cdot$ MAPK $\cdot$ Sty $1 \cdot$ Polarized growth

\section{Polarized growth in fission yeast}

A major challenge in modern biology is to understand how cellular components sense size, measure distances, and position themselves at specific locations inside the cell, so that the cell establishes distinct functional domains to generate polarized growth. Cell polarization can occur in response to extracellular or intracellular signals, or even without spatial cues (WedlichSoldner and Li 2003; Martin 2015). Polarization requires the

Pilar Pérez

piper@usal.es

$\triangle$ Jose Cansado jcansado@um.es

1 Instituto de Biología Funcional y Genómica, Consejo Superior de Investigaciones Científicas and Universidad de Salamanca, 37007 Salamanca, Spain

2 Yeast Physiology Group, Department of Genetics and Microbiology, Facultad de Biología, Universidad de Murcia, 30071 Murcia, Spain local concentration of activators that enhance signaling at a specific point and global inhibitors that favor competition for the activation at different zones. The main elements necessary to generate and maintain cell polarity include the polarity markers, which are proteins or lipids that mark the points where polarized cell growth will be established, and the cytoskeleton, which directs the arrival of the secretion vesicles towards the zone of active growth (Huisman and Brunner 2011).

Schizosaccharomyces pombe is an attractive model for studying polarized growth due to its simple genetic manipulation and because it undergoes marked changes in the growth pattern throughout its life cycle (Hoffman et al. 2015). $S$. pombe has a cylindrical morphology and maintains a constant diameter of about 3-4 $\mu \mathrm{m}$ throughout the cell cycle and is divided by fission in its middle zone through the formation of a septum. The cells have a length of 7-8 $\mu \mathrm{m}$ at the beginning of the cell cycle and grow apically. During G1, only the pole that already existed in the previous cell (old end) grows. Later, at the beginning of $\mathrm{G} 2$, the end formed in the previous division (new end) initiates growth in a process known as 
NETO (new end takes off), and the cells maintain bipolar growth until they reach $13-15 \mu \mathrm{m}$, at the time of cellular division. Then, cells enter mitosis and stop growth at the tips. At the end of mitosis, an actomyosin ring forms which will contract with the simultaneous deposition of membrane and cell wall material, called the septum that divides the cell in two. A partial and controlled digestion of the cell wall formed separates the two daughter cells (Garcia Cortes et al. 2016) (Fig. 1). During vegetative growth, fission yeast has cytoplasmic microtubules organized in 2 to 6 antiparallel bundles along the longitudinal axis of the cell. The minus ends (-) of the microtubules are anchored to the nuclear envelope, and the plus (+) ends grow towards both cell poles where microtubules rapidly depolymerize. The forces exerted by the growing end of the microtubule on contacting the poles of the cell contribute to the correct positioning of the nucleus (Tran et al. 2001) which subsequently determines the site of cell division.

At the plus end of the microtubules, a group of highly conserved proteins called + TIPs accumulates. TIPs are involved in the spatial and temporal control of microtubule dynamics and orientation along the longitudinal axis of the cell and include the EB1 protein Mal3, the kinesin Tea2, and the CLIP-170 protein family Tip1 (Huisman and Brunner 2011). Additionally, the microtubules transport and deposit Teal and Tea4 proteins at the cell poles. Tea1 is a protein with Kelch repeats, and Tea4, a protein with a SH3 domain. Once in the pole membrane, Tea1 and Tea4 remain localized to both cell ends along the cell cycle acting as polarity markers. Accordingly, cells lacking Tea1 or Tea4 show a curved and/or branched morphology and a NETO defect, suggesting that besides being important for the selection of the growth site, Teal and Tea4 also play a role during the transition to bipolar growth (Mata and Nurse 1997; Martin et al. 2005; Tatebe et al. 2005).

The relationship between microtubules and cell morphology includes a feedback loop, in which the microtubules maintain the cylindrical shape of the cell controlling the positioning of the polarized growth zones while, in turn, the cylindrical shape controls the microtubule organization (Terenna et al. 2008).

It is not completely known how the Tea1-Tea 4 complex is anchored to the membrane of the cell poles, although Mod5 and Tea3 proteins participate in the polarized location of Tea1. Mod5 is a prenylated protein that may favor the formation of polymerized Teal nodes (Bicho et al. 2010). Tea3 is structurally related to Tea1 and might participate in its localization at the non-growing pole (Snaith et al. 2005).

Another polarity marker is Pom1, a member of the DYRK family of protein kinases whose localization requires the microtubule cytoskeleton and the Tea1-Tea 4 complex. Pom1 absence produces similar defects to those observed in the cells lacking Tea1 or Tea4, in the positioning of the growth site and in NETO, and also produces defects of cytokinesis (Bähler and Pringle 1998). Pom1 forms a gradient in the membrane that extends from the poles to the center of the cell and acts as a dose-dependent inhibitor of entry into mitosis. It has been proposed that when the cell grows, the levels of Pom1 in the center of the cell decrease, allowing the inhibition of the kinase Wee1. Thus, Pom1 gradients may control cell length and entry into mitosis (Martin and Berthelot-Grosjean 2009;

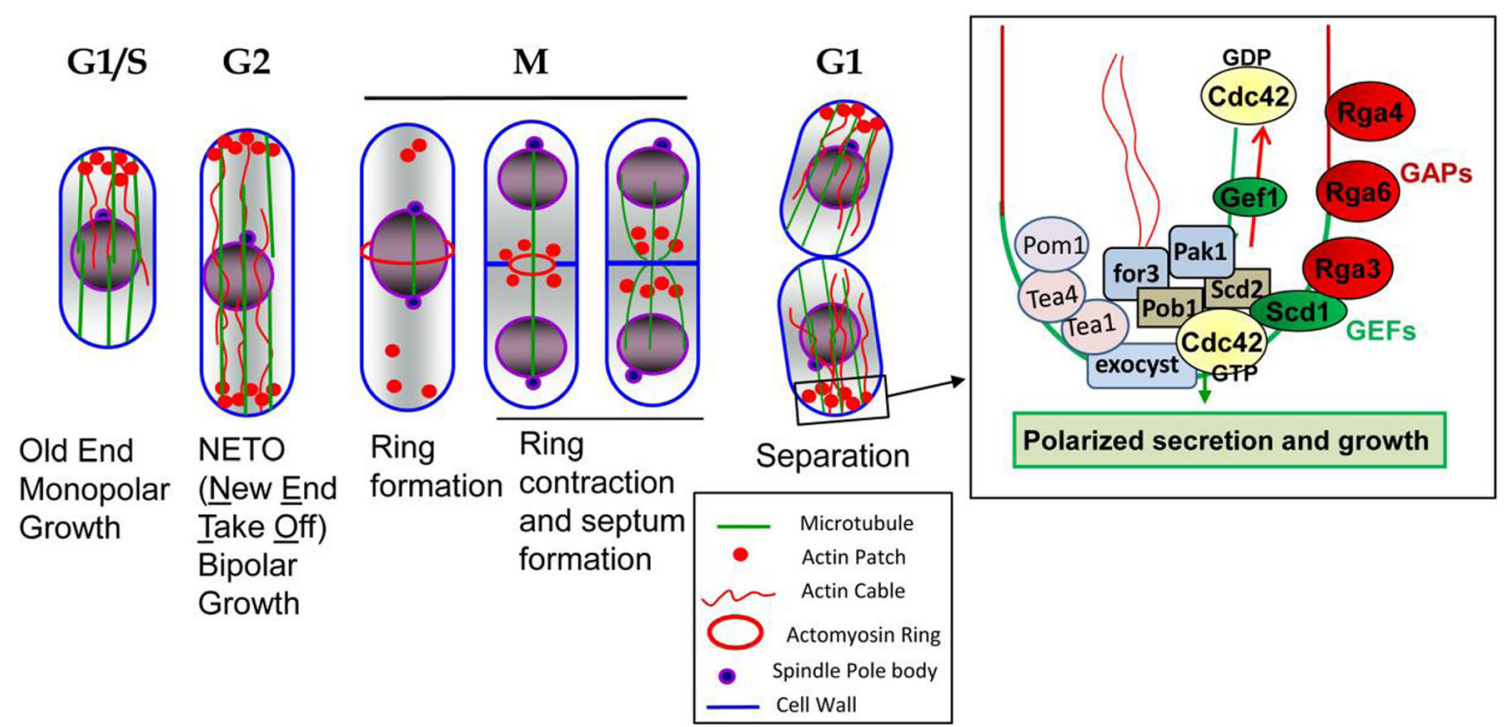

Fig. 1 Schematic representation of $S$. pombe vegetative cycle and polarized growth. Fission yeast cell cycle has a short G1 and S phases that mainly occur at the end of cytokinesis and cell separation, so daughter cells quickly reach G2. Initially, cells grow at the old end (opposite to the division site). During early G2, NETO occurs and cells grow at both poles until mitosis, when polar grow stops and cytokinesis and septation occur at the middle of the cells. Microtubules and actin rearrangements during cell cycle are shown. The inset to the right shows the cortical localization of some polarity players. The Tea1-Tea4 complex is deposited by microtubules at cell ends, where it binds to the formin For 3 which is recruited and activated by Cdc42-Pob1. Scd1-Scd2 complex is bound to $\mathrm{Cdc} 42$ at the growing poles. Active $\mathrm{Cdc} 42$ signals to its targets For3, Pak1, and the exocyst, while the GAPs (Rga4, Rga6, Rga3) restrict the activity of $\mathrm{Cdc} 42$ to the tip 
Moseley et al. 2009). However, this last role has been subsequently questioned (Wood and Nurse 2013). What remains accepted is the role of Pom1 in cell polarity, since its function is critical to mark the identity of the poles as growth zones (Tatebe et al. 2008), and preventing cytokinesis to occur there (Huang et al. 2007). Tea4 acts as a regulatory subunit of the PP1 phosphatase that recruits its catalytic subunit Dis2 to the cell poles (Alvarez-Tabarés et al. 2007). The Tea4-Dis2 complex then promotes local dephosphorylation of Pom1, which exposes its membrane binding region (Kokkoris et al. 2014).

Tea1, Tea4, and Pom1 can regulate the NETO process through its interaction with For3, a formin that is involved in the nucleation of interphase actin cables. For 3 interacts with Tea4, and its presence in the new pole seems to provide the activity necessary to induce bipolar growth (Martin et al. 2005). On the other hand, For3 localization and activation require the active GTPase $\mathrm{Cdc} 42$ and the actin nucleationpromoting factor Bud6 (Martin et al. 2007). Additionally, the kinase Pom1 could participate in NETO by regulating the localization of the protein Rga4, which negatively regulates the GTPase Cdc42. Rga4 is located on the sides of the cell and in the division zone at the end of cytokinesis. After the separation of the cells, Rga4 remains in the non-growing new pole, and its exclusion from the growing pole requires Pom 1 (Tatebe et al. 2008).

While $S$. pombe microtubule cytoskeleton is required to define the growth area, it is not necessary for polarity maintenance, which is intimately linked to the distribution of the actin cytoskeleton. In S. pombe, as in other fungi, actin is organized in three distinct structures: actin patches, actin cables, and actomyosin contractile ring (Kovar et al. 2011) (Fig. 1). Additionally, during the conjugation process, an actin structure called "actin fusion focus" is formed (Dudin et al. 2017). The proteins responsible for the nucleation of the actin filaments are the Arp2/3 protein complex and the formins (Pollard 2007). S. pombe possesses an Arp2/3 protein complex (Winter et al. 1997), and three formins, For3, Cdc12, and Fus1 which nucleate actin filaments (Pollard 2007). Actin patches are nucleated by the Arp2/3 complex. They are dense networks of branched actin filaments required for endocytosis, and their distribution coincides with polarized growth regions: the poles, in interphase cells, and the central region during cell division (Gachet and Hyams 2005). Interphase actin cables are nucleated by the formin For3. They are polarized bundles of parallel actin filaments distributed along the longitudinal axis of the cells and are involved in the transport of secretory vesicles towards the polarized growth zones. The actin that forms the actomyosin ring is mainly nucleated by the Cdc12 formin (Yonetani and Chang 2010), although For3 also has a minor role (Coffman et al. 2013). The formin Fus1 forms the "actin fusion focus" during the process of conjugation (Dudin et al. 2017).

\section{Fission yeast Rho GTPases}

The Rho family of GTPases constitutes a subgroup of the Ras superfamily of the small GTPases which is present in all eukaryotic organisms from yeasts to humans. Rho GTPases are known to regulate cytoskeletal rearrangements, cell polarity, cell motility, axon guidance, vesicle trafficking, and the cell cycle (Hodge and Ridley 2016). Ras and Rho GTPases are $20-40 \mathrm{kDa}$ monomeric proteins that essentially function as molecular switches because they bind guanine nucleotides, and their conformation changes depending on the nucleotide to which they are attached. They switch between an inactive conformation when bound to GDP, and an active conformation when bound to GTP that allows the interaction with their effector proteins (Vetter and Wittinghofer 2001). These GTPases exert their functions bound to the membranes and must be post-traslationally modified by (a) covalent addition of a farnesyl or geranylgeranyl group to a cysteine residue located at the conserved $\mathrm{C}$-terminal end of the protein called the CaaX box, (b) proteolytic cleavage of the tripeptide aaX by a conserved endopeptidase located in the endoplasmic reticulum, and (c) methylation of the free carboxyl group of the cysteine by an evolutionarily conserved isoprenylcysteine-Ocarboxyl methyltransferase, which also resides in the endoplasmic reticulum (Wennerberg et al. 2005). Some Rho GTPases may also undergo palmitoylation, a reversible process that increases their interaction with the membranes and enhances signaling (Aicart-Ramos et al. 2011).

In general, small GTPases show a high binding affinity for both GDP and GTP. Consequently, although the cellular concentration of GTP is higher than that of GDP, the exchange of GDP by GTP is very ineffective. On the other hand, the intrinsic GTPase activity of Rho proteins which hydrolyzes GTP to GDP is very low. To achieve the cycling between active and inactive conformation and perform their functions correctly, small GTPases are regulated by activating proteins called GEFs (guanine nucleotide exchange factors) and by inactivating proteins called GAPs (GTPase activating proteins). GEFs decrease the binding affinity of the nucleotides, favoring the exchange between GDP and GTP, and GAPs accelerate the hydrolysis of the bound GTP. GEFs and GAPs are proteins with multiple domains that permit the binding to the membranes and to other proteins. Therefore, in addition to regulating the activation status of the GTPases, GEFs and GAPs help regulate their location and serve as a scaffolding proteins that allow the formation of signaling protein complexes (Bos et al. 2007). Some Rho GTPases are also regulated by GDIs (GDP dissociation inhibitors), which mask the isoprenoid group and remove the GTPases from the membrane (Tiedje et al. 2008).

In the fungal kingdom, Rho GTPases are key regulators of cell morphogenesis and polarity. In these organisms, polarized cell growth requires the simultaneous addition of new plasma 
membrane and synthesis of new cell wall in the growing area. The secretory pathway must provide precursors, new membranes, and the wall biosynthetic enzymes at discrete areas of the cell. Rho GTPases provide the coordinated regulation of cell wall biosynthetic enzymes, actin cytoskeleton, and polarized secretion, which is necessary to maintain viability and integrity during fungal growth (Cabib et al. 1998; Perez and Rincon 2010).

The fission yeast genome codes for six Rho GTPases, named Rho1 to 5 and Cdc42, show 59 to $91 \%$ of identity at the amino acid sequence. Similar to the $S$. cerevisiae counterparts, Rho1 and Cdc42 are essential GTPases.

Rhol directly activates the enzyme responsible for the synthesis of (1-3) $\beta$-glucan, the main cell wall polymer in most fungi (Cabib et al. 1998). Fission yeast Rhol shows $73 \%$ and $67 \%$ identity with S. cerevisiae Rho1 and Homo sapiens RhoA, respectively. It localizes to the growing tips and septum area where it directly activates the (1-3) $\beta$-glucan synthase enzyme (Arellano et al. 1996), and also interacts with the protein kinase $\mathrm{C}$ (PKC) isoforms Pck1 and Pck2, to indirectly activate cell wall $\beta$-glucan and $\alpha$-glucan synthesis (Arellano et al. 1999).

The GTPase Rho2 also interacts with Pck2 to act as a positive regulator of the $\alpha$-glucan synthase (Calonge et al. 2000) and to activate the MAPK cellular integrity pathway (CIP) (Ma et al. 2006). This signaling cascade, whose central element is the MAPK Pmk1, is involved in very diverse processes such as cell wall biosynthesis, cytokinesis, ionic homeostasis, and fusion of vacuoles (Perez and Cansado 2010). Unlike Rho2, Rho1 maintains an antagonistic relationship with the CIP; its loss of function increases Pmk1 basal activity in a Rho2 and Pck2 dependent fashion, and is detrimental for the cells (Viana et al. 2013). Remarkably, Rhol can also directly activate the CIP under certain circumstances through Pck1 and Pck2 (Garcia et al. 2009; Sanchez-Mir et al. 2014b).

S. pombe GTPases Rho3 and Rho4 are not essential and both participate in the process of cytokinesis and cell separation. They regulate the polarized secretion of hydrolytic enzymes that digest the primary septum to liberate the two daughter cells (Wang et al. 2003; Santos et al. 2005). Rho3 interacts with For3, regulates the function of the exocyst, and appears to play a general role in secretion, not only during cytokinesis (Nakano et al. 2011). Rho4 is exclusively localized to the division zone and regulates the secretion of the enzymes involved in septum degradation (Santos et al. 2003; Santos et al. 2005). Rho4 also interacts with the exocyst and is necessary for the correct localization of septins (Perez et al. 2015).

Rho5 is a non-essential GTPase similar to Rho1 that is expressed during stationary phase and participates in the formation of the spore cell wall during sporulation (Rincon et al. 2006). Deletion of either Rho4 and Rho5 causes some defects in CIP activity, suggesting a possible role of both GTPases in this signaling pathway (Doi et al. 2015).

\section{Cdc42}

The GTPase Cdc 42 is a fundamental regulator of cell polarity in all eukaryotic organisms (Etienne-Manneville 2004). The crystallographic structure of human $\mathrm{Cdc} 42$, together with the information obtained from the mutations characterized in various organisms, has allowed us to define the functional domains of the protein and the interactions with its multiple regulators and effectors. Fission yeast $\mathrm{Cdc} 42$ is an essential GTPase that participates in the establishment and maintenance of cell polarity. It localizes to all cell membranes, but when activated, it concentrates to the plasma membrane of the growth areas (Estravis et al. 2011; Bendezu et al. 2015; Estravis et al. 2017). Cdc42 diffuses laterally in the plasma membrane and accumulates locally as a consequence of the lower mobility of the GTPase in its active form. The local accumulation of less mobile active $\mathrm{Cdc} 42$ will permit the polarization of growth, both in response to extra or intracellular signals, and spontaneously, through positive and negative "feedbacks" (Bendezu et al. 2015). Therefore, the spatial control of Cdc 42 activation determines $S$. pombe morphology and is performed by the Cdc42 GEFs and GAPs (Fig. 1). Two GEFs, Scd1 and Gef1, regulate Cdc42 activation and share an overlapping essential function, since single-deletion mutants of either gene are viable, whereas the double-deletion is lethal (Coll et al. 2003). However, deletion and/or overexpression of these two GEFs have very different effects; cells lacking Scd1 are rounded and unable to conjugate, whereas those lacking Gefl only show a wild type morphology with mild cytokinesis and bipolar growth defects (Coll et al. 2003). Scd1 seems to play a prominent role in the control of cell morphology, and it has recently been proposed to act as the main Cdc42 activator at the growing areas, whereas Gef1, a cytosolic protein in unperturbed cells, associates with the plasma membrane upon stress and functions as a global Cdc 42 activator (Tay et al. 2018). The main activation module of Cdc42 in S. cerevisiae is conserved in S. pombe. The fission yeast Ras1 GTPase participates in the activation of $\mathrm{Cdc} 42$ both in the conjugation process and in the polarized growth by stabilizing Scd1 together with the adaptor protein Scd2. These proteins are homologous to $S$. cerevisiae $\mathrm{Cdc} 24$ and Bem1 respectively. Scd2 favors the interaction of Scd1 with Ras1 and Cdc42 (Chang et al. 1994), and Scd1 and Scd2 are mutually dependent for their correct localization at the growth zones (Kelly and Nurse 2011).

The kinase Orb6 from the NDR family negatively regulates the localization of Gefl at the poles and thus, the polarized activation of Cdc42. Orb6 phosphorylates Gefl, promoting its binding to the 14-3-3 protein Rad24. This protein removes Gef1 from the membrane, controlling the activation of $\mathrm{Cdc} 42$ (Das et al. 2015). The phosphatase complex formed by Tea4Dis2 and the Orb6 kinase might conversely regulate the activation of Cdc42 mediated by Gef1 (Kokkoris et al. 2014). 
Three GAPs, Rga4, Rga6, and Rga3, have been shown to inactivate Cdc42 (Tatebe et al. 2008; Revilla-Guarinos et al. 2016; Gallo Castro and Martin 2018). Cells lacking Rga4 are wider and shorter than a wild type strain, suggesting that Rga4 contributes to restricting the activity of $\mathrm{Cdc} 42$ to the cell poles (Das et al. 2007; Tatebe et al. 2008). Rga4 is located to the cell sides and is excluded from the growing poles, while Pom1 kinase is necessary for its exclusion from the pole that is not growing (Tatebe et al. 2008). Rga6 collaborates with Rga4 to restrict active Cdc42 at the cell tips and maintains cell dimensions. Cells lacking Rga6 are viable but slightly shorter and broader than wild type cells, and simultaneous absence of Rga6 and Rga4 makes rounded cells. Rga6 localizes to the plasma membrane at the cell sides, forming clusters different from those made by Rga4 (Revilla-Guarinos et al. 2016). The third Cdc42 GAP, Rga3, localizes with Cdc42-GTP to sites of polarity. Rga3 synergizes with the Cdc42 GAPs Rga4 and Rga6 to restrict Cdc42-GTP zone sizes during mitotic growth but is dispensable for cell polarization. Rga3 also regulates the unstable Cdc42-GTP patches important for the wandering motion that favors mating during sexual differentiation. In consequence, cells lacking Rga3 hyperpolarize and have defects in conjugation (Gallo Castro and Martin 2018).

Once activated, Cdc42 controls cell polarity by signaling to its effector proteins, which are the formin For3, responsible for the formation of interphase actin cables, and the kinases of the PAK family Pak1/Shk1 and Pak2/Shk2 (Pérez and Rincón 2010) (Fig. 1). In addition, Cdc42 regulates the location of the exocyst and the traffic of cellular vesicles (Estravis et al. 2011; Bendezu and Martin 2011). The polarized secretion mediated by the actin cables, together with the binding of the vesicles to the plasma membrane mediated by the exocyst, constitutes two complementary and necessary processes for polarized growth that are regulated by Cdc42 (Bendezu and Martin 2011). Actin cables, along with the class V myosin Myo52, promote the movement of the secretory vesicles towards active growth zones. In the absence of For3, the cells maintain a polarized growth, although they show an altered morphology and present a defect in NETO (Feierbach and Chang 2001). For3 has an autoinhibition mechanism that involves the interaction between its DAD and DID domains. Active Cdc42 releases the autoinhibition of For3 and is necessary both for its activation and its localization (Martin et al. 2007). Cdc42 also regulates the plasma membrane localization of the exocyst together with phosphatidylinositol 4,5-bisphosphate (PIP2) (Bendezu and Martin 2011; Estravis et al. 2011).

As in other eukaryotes, in fission yeast, Cdc42 signals to the PAK kinases. Scd 2 adaptor protein facilitates the interaction of active Cdc42 with Pak1/Shk1 (Chang et al. 1999). This kinase is essential for cell growth, and cells that carry the thermosensitive mutant allele $o r b 2-34$ are rounded, indicating that is required for polarized growth (Marcus et al. 1995). The Pak1 targets described to date include Teal (Kim et al. 2003),
Rga8 (Yang et al. 2003), the type-I myosin Myo1 associated with the actin patches (Attanapola et al. 2009), and Rlc1, the light chain of type II myosin which is part of the actomyosin ring (Loo and Balasubramanian 2008). Pak1 also regulates the activation of the MAPK pathway that signals the cellular conjugation process ( $\mathrm{Tu}$ et al. 1997).

In $S$. pombe bipolar cells, an alternance in active $\mathrm{Cdc} 42$ levels is observed over time between one pole and another. This oscillatory behavior is attributed to the existence of a regulation by positive and negative feedbacks. It has been proposed that Gefl may be part of the positive feedback, while Pak1controls the negative feedback mechanism (Das et al. 2012).

Pak2/Shk2 is not essential, and its functions are partially redundant with those of Pak1 (Sells et al. 1998). It has recently been described that Pak2 is required for a correct cell fusion during mating (Vjestica et al. 2018).

\section{The stress-activated MAP kinase (SAPK) pathway}

MAPK signaling pathways play a key role in eukaryotes to elicit proper adaptive responses to changes in the surrounding or adverse conditions (Gacto et al. 2003). The canonical threecomponent MAPK module is composed by a MAPK kinase kinase (MAPKKK), MAPK kinase (MAPKK), and MAP kinase (MAPK), which become sequentially phosphorylated in response to multiple external and internal cues. Once activated, the MAPK phosphorylates extranuclear substrates and shifts into the nucleus to phosphorylate specific transcription factors to execute a transcriptional program triggered by the stimuli (Bluthgen and Legewie 2008). S.pombe has become an excellent model organism to unveil novel mechanisms of general significance linked to MAPK-dependent signaling outputs since, in contrast to complex eukaryotes, it has only three MAPK pathways. Moreover, two of them show significant functional homology to those of higher cells (Gacto et al. 2003). They are the stress-activated (SAPK) and the cell integrity (CIP) MAPK pathways, which are homologous to mammalian p38 and ERK5 pathways, respectively (Perez and Cansado 2010). The SAPK pathway plays a critical role in fission yeast by modulating cell cycle control and the general adaptive response to stress. Indeed, mutants in this pathway display cell cycle control defects and severe growth sensitivity in response to stress. We will focus on the organization and functions of the SAPK pathway, taking into account the recent findings on its role during $S$. pombe polarized growth.

The central element of the pathway is the MAPK Sty1, a p38 ortholog that becomes activated in response to multiple cues including hyperosmotic stress, heat shock, cold stress, hydrogen peroxide, or nutrient starvation (Perez and Cansado 2010). The MAPK module is activated by the general response regulator protein Mcs4 and includes the 
redundant MAPKKKs Wak1 and Win1 and the MAPKK Wis1 (Fig. 2). The sensors involved in the detection and signaling of most of the stresses that activate the SAPK pathway are currently unknown, except during oxidative stress induced by $\mathrm{H}_{2} \mathrm{O}_{2}$. In this case, the activating signal is transmitted through a multistep phosphorelay module composed by two sensor histidine kinases (Mak2 and Mak3), the histidinecontaining phosphotransfer protein Mprl, and the response regulator Mcs4 (Fig. 2) (Buck et al. 2001; Quinn et al. 2002). In addition to its defined role during oxidative stress, Mcs4 is necessary for the activation of Sty 1 in response to any type of stress (Buck et al. 2001; Soto et al. 2002). Mcs4 stabilizes a heteromeric complex that includesWak1 and Win1 MAPKKKs, thus promoting the physical interaction with the MAPKK Wis1. Once activated by Wak1 and Win1, Wis1 binds and phosphorylates cytoplasmic Sty1 at two conserved threonine-171 and tyrosine-173 amino acids within its activation loop (-TGY- motif) (Nguyen et al. 2002). Therefore, the Mcs4-MAPKKKs complex may act as a stabilizing platform that modulates the correct signaling through the SAPK pathway in response to stress (Morigasaki et al. 2013).
Once phosphorylated, Sty1 translocates to the cell nucleus to associate with and phosphorylate Atf1, a transcription factor with a cAMP-response element (CRE)-binding motif that is ortholog to mammalian ATF-2 (Shiozaki and Russell 1996; Wilkinson et al. 1996). Then, phosphorylated Atf1 induces the expression of a group of genes including the core environmental stress response (CESR) genes, which participate in the adaptive cell response against stress (Chen et al. 2003). Accordingly, S. pombe mutants lacking Atfl show growth sensitivity to stress quite similar to that of cells lacking Sty1. It was originally described that the phosphorylation of Atfl by Sty1 increases the stability of the transcription factor (Lawrence et al. 2007). However, recent evidences employing multiple Atf1 phospho-mutants have shown that Sty-mediated Atf1 phosphorylation does not stabilize the factor nor does it stimulate its binding to DNA (Salat-Canela et al. 2017). Rather, it provides a structural platform of interaction with the transcriptional machinery to facilitate transcription initiation and the massive gene expression shift allowing cellular adaptation to stress (Salat-Canela et al. 2017).

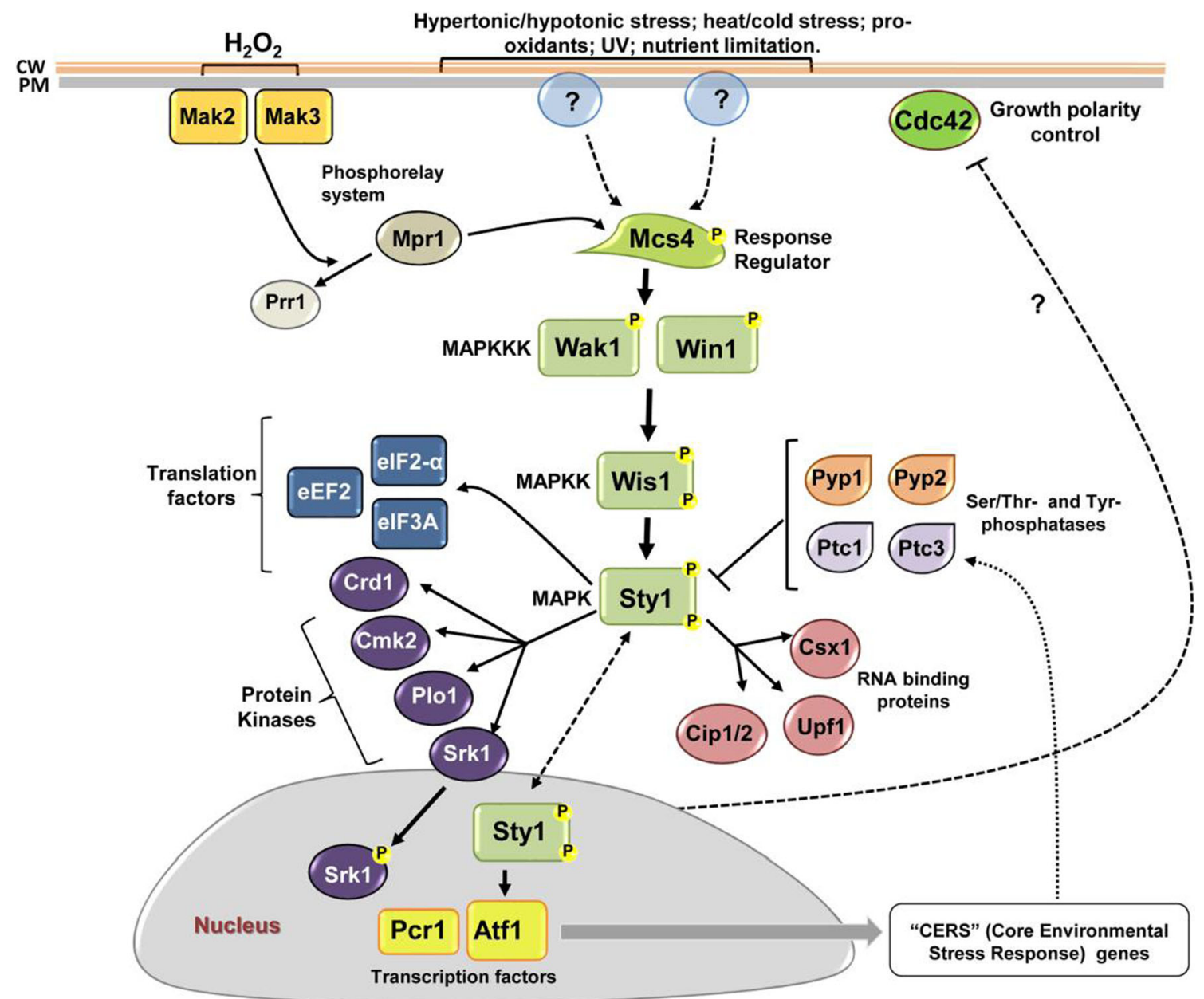

Fig. 2 The core components of the stress-activated MAPK pathway in the fission yeast. See text for specific details 
The tyrosine phosphatases Pyp1 and Pyp2 and the serine/ threonine phosphatases of the PP2C family Ptc1 and Ptc3 dephosphorylate activated Sty1 in vivo (Fig. 2). Pyp1 is responsible for Sty 1 deactivation during unperturbed vegetative growth, while both Pyp1 and Pyp2 are involved in MAPK dephosphorylation during stress (Millar et al. 1995). Ptc1 and Ptc3 are particularly relevant during the recovery of basal Sty1 activity after a thermal shock (Nguyen and Shiozaki 1999). Besides Atf1, several RNA binding proteins (Csx1, Upf1, Cip1, and Cip2) are relevant effectors of Sty1. These proteins are essential for the accurate control of both gene expression and cellular response to oxidative stress (Rodriguez-Gabriel et al. 2003; Martin et al. 2006). Sty1 also phosphorylates several translational regulatory factors (eIF $2 \alpha$, eEF2, eIF3a) (Asp et al. 2008; Berlanga et al. 2010), suggeting a key role for the fission yeast SAPK pathway in the translational initiation and adaptation under stress. The MAPKactivated protein kinases (MAPKAP) $\mathrm{Cmk} 2$ and Srk1 are also phosphorylated by Sty1. Cmk2 kinase is necessary to overcome the stress caused by metalloid agents, in particular arsenite. This control is exerted by negatively regulating translation through $\mathrm{Cpc} 2$, the RACK1 ortholog in fission yeast (Sanchez-Marinas et al. 2018). Srk1 regulates the onset of mitosis by phosphorylating and inhibiting Cdc25 phosphatase in response to non-genotoxic stresses such as osmotic or oxidative stress (Lopez-Aviles et al. 2005). Finally, Sty1 regulates mitotic commitment and recovery from stress through the indirect phosphorylation of polo kinase Plo1 (Petersen and Hagan 2005). Additionally, osmotic stress induces Sty1mediated hyperphosphorylation of the kinase Cdr1 and cells delay division. $\mathrm{Cdr} 1$ is a mitotic inducer that promotes mitotic entry by inhibiting Weel (Opalko and Moseley 2017).

\section{Crosstalk between Cdc42 and the SAPK pathway during fission yeast polarized growth}

Rho1 and Rho2 GTPases are regarded as true upstream activators of the cell integrity MAPK pathway (CIP) in response to multiple stimuli (Garcia et al. 2009; Sanchez-Mir et al. 2014a, b). In contrast, there was no knowledge of possible crosstalk mechanisms between fission yeast Rho GTPases and the SAPK pathway until very recently (see below). Likely due to the fact that, except for the oxidative stress induced by hydrogen peroxide (Buck et al. 2001; Quinn et al. 2002), no specific sensors have been described that activate the SAPK pathway in response to other stimuli (hyperosmotic stress, nutrient limitation, heavy metals, or pro-oxidants) (Perez and Cansado 2010). Information on the functional interaction of fission yeast MAPKs with cell polarity control proteins is also quite limited. An early report described that the polarity marker Tea4 associates in vivo with the SAPK
MAPKKK Win1 (Tatebe et al. 2005). In agreement with this observation, mutants lacking either Tea4 or Teal display severe cell-polarity defects in response to stimuli like osmostress or heat-stress that activate the SAPK pathway. These observations suggest that fission yeast SAPK pathway might play a prominent role together with Tea4 and Tea1 to establish cell polarity under stress conditions (Tatebe et al. 2005). Osmotic stress arrests polarized growth, leads to dissociation of F-actin patches from the cell tips, and "freezes" the interphase microtubules before return to a dynamic behavior (Robertson and Hagan 2008). Notably, the time needed for microtubules to resume normal dynamics is positively controlled by the SAPK pathway via Atf1-mediated transcription, and is totally independent of CIP signaling (Robertson and Hagan 2008). Other Sty1-activating stresses like heat or centrifugation also lead to a transient dispersal of F-actin patches from cell tips, and Sty1-mediated indirect phosphorylation of serine 402 of the polo kinase Plo1 is required for the recruitment of patches back to cell tips (Petersen and Hagan 2005; Soto et al. 2007). However, the finding that the kinetics of tip growth following osmotic stress remains unaffected in Plo1-S402A/E mutants suggests that the role of the SAPK pathway to promote resumption of tip growth after osmotic stress is independent of Plo1mediated signaling (Robertson and Hagan 2008).

Remarkably, a recent study revealed the existence of a functional interaction between Cdc42 GTPase and the SAPK MAPK Sty1 during fission yeast polarized growth (Mutavchiev et al. 2016) (Fig. 3). Treatment of exponentially growing $S$. pombe cells with the sponge-derived macrolide Latrunculin A (LatA), which prevents F-actin cytoskeleton assembly, activates Sty1, promotes the dispersal of active $\mathrm{Cdc} 42$ from the cell tips, and fosters the cessation of polarized growth (Mutavchiev et al. 2016). Importantly, loss of Sty1 MAP kinase signaling prevents latrunculin A-induced dispersal of the active Cdc42 module and allows polarized growth in the absence of the actin cytoskeleton (Mutavchiev et al. 2016). The role of Sty1 to promote Cdc42 dispersal is independent of stress-induced gene expression via Atfl or Plo1-mediated regulation of cell polarity, since LatA treatment still led to the dispersal of Cdc42 in Atfl-null cells, as well as in Plo1-402A, Plo1-S402E, and analog-sensitive Plo1 mutants (Mutavchiev et al. 2016). Most important, Sty1 activation in the absence of any external stress is sufficient to disperse the Cdc42 module from cell tips in unperturbed cells, suggesting that fission yeast Sty1 is an important physiological regulator of the $\mathrm{Cdc} 42$ polarity module (Mutavchiev et al. 2016). However, the underlying mechanism/s involved in this control is/are currently unknown. $\mathrm{Cdc} 42$ amino acid sequence harbors a single consensus site for MAPK phosphorylation, and Sty1 might directly associate to $\mathrm{Cdc} 42$ and negatively regulate its activity but no study has shown Sty1 to localize at cell membranes and/or poles. Likely, Sty1 control of Cdc42 functions is exerted indirectly through one or several 
Fig. 3 Active $\mathrm{Cdc} 42$ depolarization in response to the activation of the SAPK MAPK Sty1. Fluorescence micrographs of the CRIB-GFP probe to visualize active Cdc42. GTP-bound $\mathrm{Cdc} 42$ localizes to the cell growing poles in wild type and $\operatorname{sty} 1 \Delta$ fission yeast cells (arrows). Upon $\mathrm{F}$-actin stress induced with Latrunculin A treatment during 20 min, active $\mathrm{Cdc} 42$ becomes depolarized (asterisks) in wild type cells, but remains highly polarized in sty $1 \Delta$ cells as described in (Mutavchiev et al. 2016)

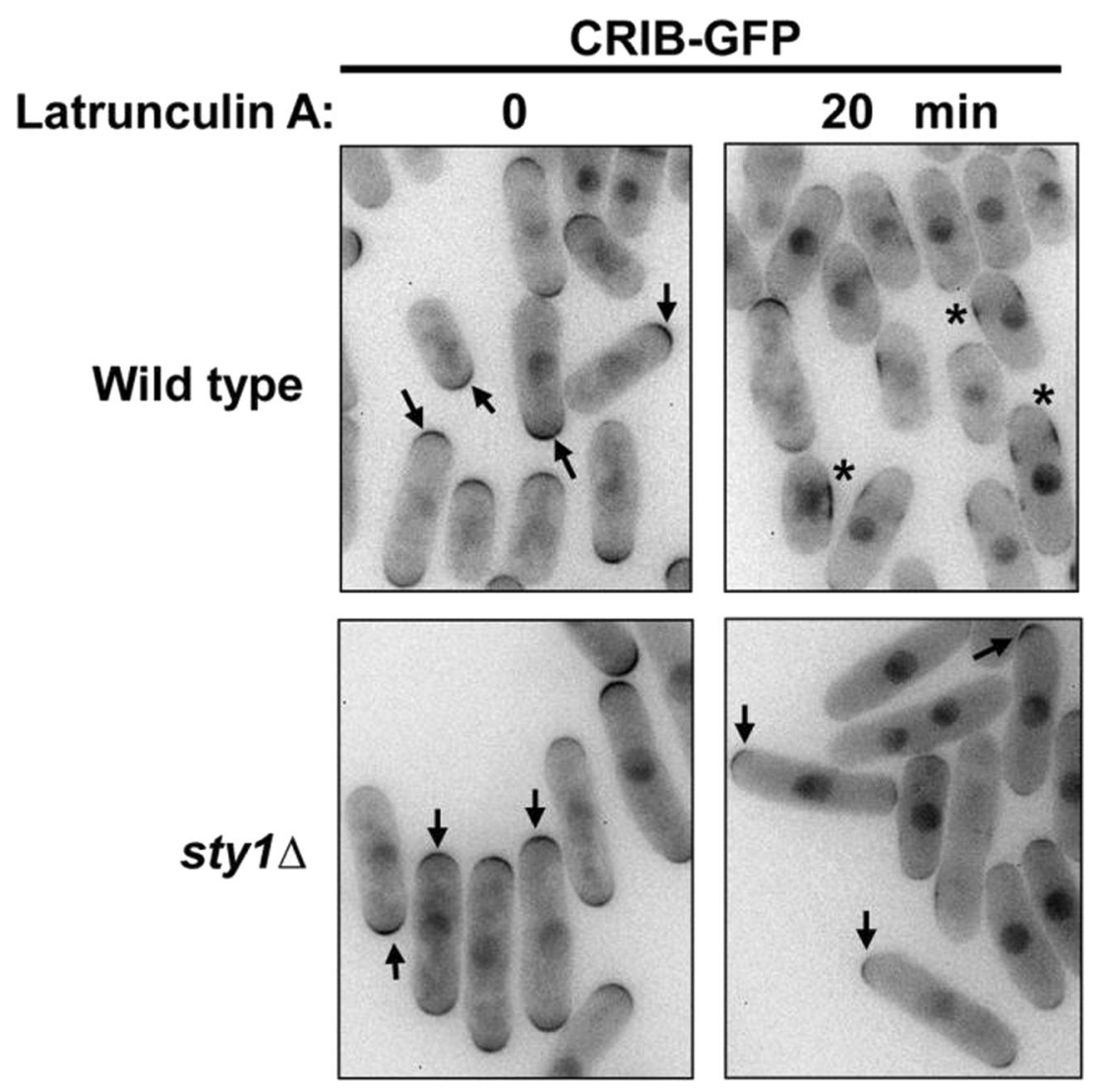

regulators of the Cdc42 activation threshold, including GEFs (Gef1, Scd1) and/or GAPs (Rga4, Rga6, and Rga3). The finding that recovery of active $\mathrm{Cdc} 42$ at the cell tips after Sty1 inactivation is very rapid (Mutavchiev et al. 2016) also suggests that the MAP kinase may negatively regulate the coupling of the Cdc42 module to cell-polarity landmarks like Tea1-Tea4 or to the formin For3. In this context, reducing the growth rate of fission yeast cells using chemical, genetic, and mechanical means has been shown to induce active Cdc42 domains to rapidly oscillate from cell tips around the cell surface, whereas an abrupt increase in growth rate improves polar domain stabilization (Haupt et al. 2018). A candidate screen for suppressors of the detachment of active Cdc42 domains from cell tips in response to osmotic stress revealed that vesicular transport along actin cables mediated by For3 and the myosin type V Myo52 are important players in mediating this process (Haupt et al. 2018). Remarkably, Sty1-less cells exhibited a lower suppression of this phenotype, which might suggest a functional interaction between Sty1 and For3. However, active Cdc42 domains remained fully polarized during osmotic stress in cells lacking both Sty1 and For3 as compared to the single Sty1 and For3deleted mutants, suggesting that actin cable-mediated transport (For3) and the SAPK pathway (Sty1) act independently during this response (Haupt et al. 2018). The positive feedback between growth and polarity that allows cells to dynamically reorient their growth axis towards empty spaces is conserved in distant filamentous fungi, and it might represent a basal property of eukaryotic polarization in response to environmental changes.

\section{Concluding remarks}

Diverse studies have revealed the role of MAPK signaling during cell polarization in complex eukaryotes. S. pombe has greatly contributed to this research area, particularly with regard to growth site positioning and the key role of the evolutionary conserved Rho GTPase Cdc42. However, understanding the mutual and functional relationships between MAPK cascades and the cell polarization machinery remains an important question in the field. Future work using this fission yeast as a model will undoubtedly help to provide a mechanistic understanding of the regulation of cell polarity by the stress-activated MAPK cascades, to identify the specific targets involved in these complex mechanisms, and to unveil their biological significance during cellular adaptation to environment.

Acknowledgments We thank D. M. Posner for language revision. This work was supported by grants BIO2015-69958-P and BFU2017-82423-P from the Ministry of Science, Innovation and Universities (MICIU) and 
grants CSI068P17 and Escalera de Excelencia CLU-2017-03 from the Regional Government of Castilla y León (JCyL/FEDER) and the European Regional Development Fund (ERDF). The authors wish to acknowledge the many other studies on fission yeast cell polarity and apologize for not citing every important contribution in this review due to space limitations.

\section{Compliance with ethical standards}

Conflict of interest The authors declare that they have conflict of interest.

\section{References}

Aicart-Ramos C, Valero RA, Rodriguez-Crespo I (2011) Protein palmitoylation and subcellular trafficking. Biochim Biophys Acta 1808:2981-2994

Alvarez-Tabarés I, Grallert A, Ortiz JM, Hagan IM (2007) Schizosaccharomyces pombe protein phosphatase 1 in mitosis, endocytosis and a partnership with Wsh3/Tea4 to control polarised growth. J Cell Sci 120:3589-3601

Arellano M, Durán A, Pérez P (1996) Rho 1 GTPase activates the (13)beta-D-glucan synthase and is involved in Schizosaccharomyces pombe morphogenesis. EMBO J 15:4584-4591

Arellano M, Valdivieso MH, Calonge TM, Coll PM, Duran A, Perez P (1999) Schizosaccharomyces pombe protein kinase C homologues, pck $1 \mathrm{p}$ and pck $2 \mathrm{p}$, are targets of rho1p and rho2p and differentially regulate cell integrity. J Cell Sci 112:3569-3578

Asp E, Nilsson D, Sunnerhagen P (2008) Fission yeast mitogen-activated protein kinase Sty1 interacts with translation factors. Eukaryot Cell 7:328-338

Attanapola SL, Alexander CJ, Mulvihill DP (2009) Ste20-kinasedependent TEDS-site phosphorylation modulates the dynamic localisation and endocytic function of the fission yeast class I myosin, Myo1. J Cell Sci 122:3856-3861

Bähler J, Pringle JR (1998) Pom1p, a fission yeast protein kinase that provides positional information for both polarized growth and cytokinesis. Genes Dev 12:1356-1370

Bendezu FO, Martin SG (2011) Actin cables and the exocyst form two independent morphogenesis pathways in the fission yeast. Mol Biol Cell 22:44-53

Bendezu FO, Vincenzetti V, Vavylonis D, Wyss R, Vogel H, Martin SG (2015) Spontaneous Cdc42 polarization independent of GDImediated extraction and actin-based trafficking. PLoS Biol 13: e1002097

Berlanga JJ, Rivero D, Martin R, Herrero S, Moreno S, de Haro C (2010) Role of mitogen-activated protein kinase Sty1 in regulation of eukaryotic initiation factor 2alpha kinases in response to environmental stress in Schizosaccharomyces pombe. Eukaryot Cell 9:194-207

Bicho CC, Kelly DA, Snaith HA, Goryachev AB, Sawin KE (2010) A catalytic role for Mod5 in the formation of the Teal cell polarity landmark. Curr Biol 20:1752-1757

Bluthgen N, Legewie S (2008) Systems analysis of MAPK signal transduction. Essays in Biochem 45:95-107

Bos JL, Rehmann H, Wittinghofer A (2007) GEFs and GAPs: critical elements in the control of small G proteins. Cell 129:865-877

Buck V, Quinn J, Soto Pino T, Martin H, Saldanha J, Makino K, Morgan BA, Millar JB (2001) Peroxide sensors for the fission yeast stressactivated mitogen-activated protein kinase pathway. Mol Biol Cell 12:407-419

Cabib E, Drgonová J, Drgon T (1998) Role of small G proteins in yeast cell polarization and wall biosynthesis. Annu Rev Biochem 67:307-333
Calonge TM, Nakano K, Arellano M, Arai R, Katayama S, Toda T, Mabuchi I, Perez P (2000) Schizosaccharomyces pombe rho2p GTPase regulates cell wall alpha-glucan biosynthesis through the protein kinase pck2p. Mol Biol Cell 11:4393-4401

Chang EC, Barr M, Wang Y, Jung V, Xu HP, Wigler MH (1994) Cooperative interaction of $\mathrm{S}$. pombe proteins required for mating and morphogenesis. Cell Cycle 79:131-141

Chang E, Bartholomeusz G, Pimental R, Chen J, Lai H, Wang LH, Yang $\mathrm{P}$, Marcus S (1999) Direct binding and in vivo regulation of the fission yeast $\mathrm{p} 21$-activated kinase shk1 by the $\mathrm{SH} 3$ domain protein scd2. Mol Cell Biol 19:8066-8074

Chen D, Toone WM, Mata J, Lyne R, Burns G, Kivinen K, Brazma A, Jones N, Bahler J (2003) Global transcriptional responses of fission yeast to environmental stress. Mol Biol Cell 14:214-229

Coffman VC, Sees JA, Kovar DR, Wu JQ (2013) The formins Cdc12 and For3 cooperate during contractile ring assembly in cytokinesis. J Cell Biol 203:101-114

Coll PM, Trillo Y, Ametzazurra A, Perez P (2003) Geflp, a new guanine nucleotide exchange factor for $\mathrm{Cdc} 42 \mathrm{p}$, regulates polarity in Schizosaccharomyces pombe. Mol Biol Cell 14:313-323

Das M, Wiley DJ, Medina S, Vincent HA, Larrea M, Oriolo A, Verde F (2007) Regulation of cell diameter, For3p localization, and cell symmetry by fission yeast Rho-GAP Rga4p. Mol Biol Cell 18:2090 2101

Das M, Drake T, Wiley DJ, Buchwald P, Vavylonis D, Verde F (2012) Oscillatory dynamics of $\mathrm{Cdc} 42$ GTPase in the control of polarized growth. Science 337:239-243

Das M, Nunez I, Rodriguez M, Wiley DJ, Rodriguez J, Sarkeshik A, Yates JR 3rd, Buchwald P, Verde F (2015) Phosphorylationdependent inhibition of Cdc42 GEF Gef1 by 14-3-3 protein Rad24 spatially regulates Cdc42 GTPase activity and oscillatory dynamics during cell morphogenesis. Mol Biol Cell 26:3520-3534

Doi A, Kita A, Kanda Y, Uno T, Asami K, Satoh R, Nakano K, Sugiura R (2015) Geranylgeranyltransferase Cwg2-Rho4/Rho5 module is implicated in the Pmk1 MAP kinase-mediated cell wall integrity pathway in fission yeast. Genes Cells 20:310-323

Dudin O, Merlini L, Bendezu FO, Groux R, Vincenzetti V, Martin SG (2017) A systematic screen for morphological abnormalities during fission yeast sexual reproduction identifies a mechanism of actin aster formation for cell fusion. PLoS Genet 13:e1006721

Estravis M, Rincon SA, Santos B, Perez P (2011) Cdc42 regulates multiple membrane traffic events in fission yeast. Traffic 12:1744-1758

Estravis M, Rincon SA, Portales E, Perez P, Santos B (2017) Cdc42 activation state affects its localization and protein levels in fission yeast. Microbiology

Etienne-Manneville S (2004) Cdc42-the Centre of polarity. J Cell Sci 117:1291-1300

Feierbach B, Chang F (2001) Roles of the fission yeast formin for $3 p$ in cell polarity, actin cable formation and symmetric cell division. Curr Biol 11:1656-1665

Gachet Y, Hyams JS (2005) Endocytosis in fission yeast is spatially associated with the actin cytoskeleton during polarised cell growth and cytokinesis. J Cell Sci 118:4231-4242

Gacto M, Soto T, Vicente-Soler J, Villa T, Cansado J (2003) Learning from yeasts: intracellular sensing of stress conditions. Int Microbio $16: 211-219$

Gallo Castro D, Martin SG (2018) Differential GAP requirement for Cdc42-GTP polarization during proliferation and sexual reproduction. J Cell Biol 217:4215-4229

Garcia Cortes JC, Ramos M, Osumi M, Perez P, Ribas JC (2016) The cell biology of fission yeast Septation. Microbiol Mol Biol Rev 80:779-791

Garcia P, Tajadura V, Sanchez Y (2009) The Rholp exchange factor Rgflp signals upstream from the Pmk1 mitogen-activated protein kinase pathway in fission yeast. Mol Biol Cell 20:721-731 
Haupt A, Ershov D, Minc N (2018) A positive feedback between growth and polarity provides directional persistency and flexibility to the process of tip growth. Curr Biol 28:3342-3351 e3343

Hodge RG, Ridley AJ (2016) Regulating Rho GTPases and their regulators. Nat Rev Mol Cell Biol 17:496-510

Hoffman CS, Wood V, Fantes PA (2015) An ancient yeast for young geneticists: a primer on the Schizosaccharomyces pombe model system. Genetics 201:403-423

Huang Y, Chew TG, Ge W, Balasubramanian MK (2007) Polarity determinants Tea1p, Tea4p, and Pom1p inhibit division-septum assembly at cell ends in fission yeast. Dev Cell 12:987-996

Huisman SM, Brunner D (2011) Cell polarity in fission yeast: a matter of confining, positioning, and switching growth zones. Semin Cell Dev Biol 22:799-805

Kelly FD, Nurse P (2011) Spatial control of Cdc42 activation determines cell width in fission yeast. Mol Biol Cell 22:3801-3811

Kim H, Yang P, Catanuto P, Verde F, Lai H, Du H, Chang F, Marcus S (2003) The kelch repeat protein, Tea1, is a potential substrate target of the p21-activated kinase, Shk1, in the fission yeast, Schizosaccharomyces pombe. J Biol Chem 278:30074-30082

Kokkoris K, Gallo Castro D, Martin SG (2014) The Tea4-PP1 landmark promotes local growth by dual Cdc42 GEF recruitment and GAP exclusion. J Cell Sci 127:2005-2016

Kovar DR, Sirotkin V, Lord M (2011) Three's company: the fission yeast actin cytoskeleton. Trends Cell Biol 21:177-187

Lawrence CL, Maekawa H, Worthington JL, Reiter W, Wilkinson CR, Jones N (2007) Regulation of Schizosaccharomyces pombe Atf1 protein levels by Sty1-mediated phosphorylation and heterodimerization with Pcr1. J Biol Chem 282, United States: $5160-5170$

Loo TH, Balasubramanian M (2008) Schizosaccharomyces pombe Pakrelated protein, Pak1p/Orb2p, phosphorylates myosin regulatory light chain to inhibit cytokinesis. J Cell Biol 183:785-793

Lopez-Aviles S, Grande M, Gonzalez M, Helgesen AL, Alemany V, Sanchez-Piris M, Bachs O, Millar JB, Aligue R (2005) Inactivation of the $\mathrm{Cdc} 25$ phosphatase by the stress-activated Srk1 kinase in fission yeast. Mol Cell 17:49-59

Ma Y, Kuno T, Kita A, Asayama Y, Sugiura R (2006) Rho2 is a target of the farnesyltransferase Cpp1 and acts upstream of Pmk1 mitogenactivated protein kinase signaling in fission yeast. Mol Biol Cell 17: 5028-5037

Marcus S, Polverino A, Chang E, Robbins D, Cobb MH, Wigler MH (1995) Shk1, a homolog of the Saccharomyces cerevisiae Ste20 and mammalian p65PAK protein kinases, is a component of a Ras/ $\mathrm{Cdc} 42$ signaling module in the fission yeast Schizosaccharomyces pombe. Proc Natl Acad Sci U S A 92:6180-6184

Martin SG (2015) Spontaneous cell polarization: feedback control of Cdc42 GTPase breaks cellular symmetry. Bioessays 37:1193-1201

Martin SG, Berthelot-Grosjean M (2009) Polar gradients of the DYRKfamily kinase Pom1 couple cell length with the cell cycle. Nature 459:852-856

Martin SG, McDonald WH, Yates JR 3rd, Chang F (2005) Tea4p links microtubule plus ends with the formin for $3 p$ in the establishment of cell polarity. Dev Cell 8:479-491

Martin V, Rodriguez-Gabriel MA, McDonald WH, Watt S, Yates JR 3rd, Bahler J, Russell P (2006) Cip1 and Cip2 are novel RNArecognition-motif proteins that counteract $\mathrm{Csx} 1$ function during oxidative stress. Mol Biol Cell 17:1176-1183

Martin SG, Rincon SA, Basu R, Perez P, Chang F (2007) Regulation of the formin for3p by cdc42p and bud6p. Mol Biol Cell 18:4155-4167

Mata J, Nurse P (1997) Teal and the microtubular cytoskeleton are important for generating global spatial order within the fission yeast cell. Cell 89:939-949

Millar JB, Buck V, Wilkinson MG (1995) Pyp1 and Pyp2 PTPases dephosphorylate an osmosensing MAP kinase controlling cell size at division in fission yeast. Genes Dev 9:2117-2130
Morigasaki S, Ikner A, Tatebe H, Shiozaki K (2013) Response regulatormediated MAPKKK heteromer promotes stress signaling to the Spc1 MAPK in fission yeast. Mol Biol Cell 24:1083-1092

Moseley JB, Mayeux A, Paoletti A, Nurse P (2009) A spatial gradient coordinates cell size and mitotic entry in fission yeast. Nature 459: $857-860$

Mutavchiev DR, Leda M, Sawin KE (2016) Remodeling of the fission yeast Cdc 42 cell-polarity module via the Sty 1 p38 stress-activated protein kinase pathway. Curr Biol 26:2921-2928

Nakano K, Toya M, Yoneda A, Asami Y, Yamashita A, Kamasawa N, Osumi M, Yamamoto M (2011) Pob1 ensures cylindrical cell shape by coupling two distinct rho signaling events during secretory vesicle targeting. Traffic 12:726-739

Nguyen AN, Shiozaki K (1999) Heat-shock-induced activation of stress MAP kinase is regulated by threonine- and tyrosine-specific phosphatases. Genes Dev 13:1653-1663

Nguyen AN, Ikner AD, Shiozaki M, Warren SM, Shiozaki K (2002) Cytoplasmic localization of Wis1 MAPKK by nuclear export signal is important for nuclear targeting of Spc1/Sty1 MAPK in fission yeast. Mol Biol Cell 13:2651-2663

Opalko HE, Moseley JB (2017) Dynamic regulation of Cdr1 kinase localization and phosphorylation during osmotic stress. J Biol Chem 292:18457-18468

Perez P, Cansado J (2010) Cell integrity signaling and response to stress in fission yeast. Curr Protein Pept Sci 11:680-692

Perez P, Rincon SA (2010) Rho GTPases: regulation of cell polarity and growth in yeasts. Biochem J 426:243-253

Perez P, Portales E, Santos B (2015) Rho4 interaction with exocyst and septins regulates cell separation in fission yeast. Microbiology 161: 948-959

Petersen J, Hagan IM (2005) Polo kinase links the stress pathway to cell cycle control and tip growth in fission yeast. Nature 435:507-512

Pollard TD (2007) Regulation of actin filament assembly by Arp2/3 complex and formins. Ann Rev Biophys Biomol Struct 36:451-477

Quinn J, Findlay VJ, Dawson K, Millar JB, Jones N, Morgan BA, Toone WM (2002) Distinct regulatory proteins control the graded transcriptional response to increasing $\mathrm{H}(2) \mathrm{O}(2)$ levels in fission yeast Schizosaccharomyces pombe. Mol Biol Cell 13:805-816

Revilla-Guarinos MT, Martin-Garcia R, Villar-Tajadura MA, Estravis M, Coll PM, Perez P (2016) Rga6 is a fission yeast rho GAP involved in Cdc42 regulation of polarized growth. Mol Biol Cell 27:1524-1535

Rincon SA, Santos B, Perez P (2006) Fission yeast Rho5p GTPase is a functional paralogue of Rholp that plays a role in survival of spores and stationary-phase cells. Eukaryot Cell 5:435-446

Robertson AM, Hagan IM (2008) Stress-regulated kinase pathways in the recovery of tip growth and microtubule dynamics following osmotic stress in S. Pombe. J Cell Sci 121:4055-4068

Rodriguez-Gabriel MA, Burns G, McDonald WH, Martin V, Yates JR 3rd, Bahler J, Russell P (2003) RNA-binding protein Csx1 mediates global control of gene expression in response to oxidative stress. EMBO J 22:6256-6266

Salat-Canela C, Paulo E, Sánchez-Mir L, Carmona M, Ayté J, Oliva B, Hidalgo E (2017) Deciphering the role of the signal- and Sty1 kinase-dependent phosphorylation of the stress-responsive transcription factor Atf1 on gene activation. J Biol Chem 292:1363513644

Sanchez-Marinas M, Gimenez-Zaragoza D, Martin-Ramos E, Llanes J, Cansado J, Pujol MJ, Bachs O, Aligue R (2018) Cmk2 kinase is essential for survival in arsenite by modulating translation together with RACK1 orthologue Cpc2 in Schizosaccharomyces pombe. Free Radic Biol Med 129:116-126

Sanchez-Mir L, Franco A, Martin-Garcia R, Madrid M, Vicente-Soler J, Soto T, Gacto M, Perez P, Cansado J (2014a) Rho2 palmitoylation is required for plasma membrane localization and proper signaling to the fission yeast cell integrity MAPK pathway. Mol Cell Biol 34: $2747-2759$ 
Sanchez-Mir L, Soto T, Franco A, Madrid M, Viana RA, Vicente J, Gacto M, Perez P, Cansado J (2014b) Rhol GTPase and PKC ortholog Pck1 are upstream activators of the cell integrity MAPK pathway in fission yeast. PLoS One 9:e88020

Santos B, Gutierrez J, Calonge TM, Perez P (2003) Novel rho GTPase involved in cytokinesis and cell wall integrity in the fission yeast Schizosaccharomyces pombe. Eukaryot Cell 2:521-533

Santos B, Martin-Cuadrado AB, Vazquez de Aldana CR, del Rey F, Perez $\mathrm{P}$ (2005) Rho4 GTPase is involved in secretion of glucanases during fission yeast cytokinesis. Eukaryot Cell 4:1639-1645

Sells MA, Barratt JT, Caviston J, Ottilie S, Leberer E, Chernoff J (1998) Characterization of Pak2p, a pleckstrin homology domain-containing, p21-activated protein kinase from fission yeast. J Biol Chem 273:18490-18498

Shiozaki K, Russell P (1996) Conjugation, meiosis, and the osmotic stress response are regulated by Spc1 kinase through Atf1 transcription factor in fission yeast. Genes Dev 10:2276-2288

Snaith HA, Samejima I, Sawin KE (2005) Multistep and multimode cortical anchoring of tea1p at cell tips in fission yeast. EMBO J 24:3690-3699

Soto T, Beltran FF, Paredes V, Madrid M, Millar JBA, Vicente-Soler J, Cansado J, Gacto M (2002) Cold induces stress-activated protein kinase-mediated response in the fission yeast Schizosaccharomyces pombe. Eur J Biochem 269:5056-5065

Soto T, Nunez A, Madrid M, Vicente J, Gacto M, Cansado J (2007) Transduction of centrifugation-induced gravity forces through mitogen-activated protein kinase pathways in the fission yeast Schizosaccharomyces pombe. Microbiology 153:1519-1529

Tatebe H, Shimada K, Uzawa S, Morigasaki S, Shiozaki K (2005) Wsh3/ Tea4 is a novel cell-end factor essential for bipolar distribution of Tea1 and protects cell polarity under environmental stress in S. pombe. Curr Biol 15:1006-1015

Tatebe H, Nakano K, Maximo R, Shiozaki K (2008) Pom1 DYRK regulates localization of the Rga4 GAP to ensure bipolar activation of Cdc42 in fission yeast. Curr Biol 18:322-330

Tay YD, Leda M, Goryachev AB, Sawin KE (2018) Local and global $\mathrm{Cdc} 42$ guanine nucleotide exchange factors for fission yeast cell polarity are coordinated by microtubules and the Tea1-Tea4-Pom1 axis. J Cell Sci 131. https://doi.org/10.1242/jcs.216580

Terenna CR, Makushok T, Velve-Casquillas G, Baigl D, Chen Y, Bornens M, Paoletti A, Piel M, Tran PT (2008) Physical mechanisms redirecting cell polarity and cell shape in fission yeast. Curr Biol 18:1748-1753
Tiedje C, Sakwa I, Just U, Hofken T (2008) The Rho GDI Rdil regulates Rho GTPases by distinct mechanisms. Mol Biol Cell 19:2885-2896

Tran PT, Marsh L, Doye V, Inoué S, Chang F (2001) A mechanism for nuclear positioning in fission yeast based on microtubule pushing. $\mathrm{J}$ Cell Biol 153:397-411

Tu H, Barr M, Dong DL, Wigler M (1997) Multiple regulatory domains on the Byr2 protein kinase. Mol Cell Biol 17:5876-5887

Vetter IR, Wittinghofer A (2001) The guanine nucleotide-binding switch in three dimensions. Science 294:1299-1304

Viana RA, Pinar M, Soto T, Coll PM, Cansado J, Pérez P (2013) Negative functional interaction between cell integrity MAPK pathway and Rho1 GTPase in fission yeast. Genetics 195:421-432

Vjestica A, Merlini L, Nkosi PJ, Martin SG (2018) Gamete fusion triggers bipartite transcription factor assembly to block re-fertilization. Nature 560:397-400

Wang H, Tang X, Balasubramanian MK (2003) Rho3p regulates cell separation by modulating exocyst function in Schizosaccharomyces pombe. Genetics 164:1323-1331

Wedlich-Soldner R, Li R (2003) Spontaneous cell polarization: undermining determinism. Nat Cell Biol 5:267-270

Wennerberg K, Rossman KL, Der CJ (2005) The Ras superfamily at a glance. J Cell Sci 118:843-846

Wilkinson MG, Samuels M, Takeda T, Toone WM, Shieh JC, Toda T, Millar JB, Jones N (1996) The Atf1 transcription factor is a target for the Sty1 stress-activated MAP kinase pathway in fission yeast. Genes Dev 10:2289-2301

Winter D, Podtelejnikov AV, Mann M, Li R (1997) The complex containing actin-related proteins Arp2 and Arp3 is required for the motility and integrity of yeast actin patches. Curr Biol 7:519-529

Wood E, Nurse P (2013) Pom1 and cell size homeostasis in fission yeast. Cell Cycle 12:3228-3236

Yang P, Qyang Y, Bartholomeusz G, Zhou X, Marcus S (2003) The novel Rho GTPase-activating protein family protein, Rga8, provides a potential link between Cdc42/p21-activated kinase and Rho signaling pathways in the fission yeast, Schizosaccharomyces pombe. J Biol Chem 278:48821-48830

Yonetani A, Chang F (2010) Regulation of cytokinesis by the formin cdc12p. Curr Biol 20:561-566

Publisher's note Springer Nature remains neutral with regard to jurisdictional claims in published maps and institutional affiliations. 